\title{
Genital psoriasis: a hidden multidisciplinary problem - a review of literature
}

\author{
Piotr Czuczwar ${ }^{1}$, Anna Stępniak ${ }^{1}$, Andy Goren ${ }^{2}$, Wojciech Wrona ${ }^{1}$, Tomasz Paszkowski ${ }^{1}$, \\ Mariola Pawlaczyk ${ }^{3}$, Dorota Piekarska-Myślińska ${ }^{4}$, Sławomir Woźniak ${ }^{1}$, Aldona Pietrzak ${ }^{4}$ \\ ${ }^{13 r d ~ D e p a r t m e n t ~ o f ~ G y n e c o l o g y, ~ M e d i c a l ~ U n i v e r s i t y ~ o f ~ L u b l i n, ~ P o l a n d ~}$ \\ 2University of Rome „G. Marconi", Italy \\ ${ }^{3}$ Division of Skin Diseases Prophylaxis, Deptartment of Geriatric Medicine and Gerontology, \\ Poznan University of Medical Sciences, Poland \\ ${ }^{4}$ Department of Dermatology, Venereology and Pediatric Dermatology, Medical University of Lublin, Poland
}

\begin{abstract}
Genital psoriasis is a variety of autoimmune dermatological disease - psoriasis with relapsing-remitting course, which can have an onset in all age groups. It is most often diagnosed at an advanced stage. Genital psoriasis is considered an embarrassing condition and is often misjudged as a sexually transmitted disease or allergic reaction due to low social awareness of the disease. The manifestations of genital psoriasis may differ from typical genital dermatoses and with symptoms such as itch, erythroderma and vaginal discharge may mimic other diseases at an early stage. The diagnosis and treatment of genital psoriasis may be difficult and often requires a multidisciplinary approach. The aim of this article is to present the literature review of genital psoriasis concentrating on the clinical presentation, treatment and influence on the quality of patients' life and sexual activity disorders.
\end{abstract}

Key words: psoriasis, genital psoriasis, sexual disorders, vulvar disease

\section{INTRODUCTION AND EPIDEMIOLOGY}

Psoriasis is a chronic autoimmune disease often associated with dermatitis and arthritis. The annual occurrence of psoriasis in the American population doubled between 1970 and 2000, although it may result from improved recognition of the disease [1]. There are no objective international diagnostic criteria to verify the diagnosis of psoriasis, that is based mostly on clinical observation. Relapsing-remitting course of psoriasis is another factor limiting adequate diagnosis. A European survey revealed that approximately $1 \%$ to $5 \%$ of Europeans suffer from psoriasis and $74 \%$ of psoriatic patients considered their disease as severe [2]. Psoriasis has an equal distribution between men and women. Surprisingly, 32\% of respondents reported genital psoriasis (GP) [2]. This was an unexpected result, because GP has been considered a rare manifestation of psoriasis and the literature data concerning GP are scarce. Such outcome eludes that $\mathrm{GP}$ is a more common phenomenon than it was previously thought. This is probably mainly due to patients'embarrassment about discussing the intimate genital area, also the majority of subjects do not know which specialist to choose for the best advice. General practitioners, dermatologists, gynaecologists, urologists and paediatricians are the most common choice. The cooperation between patients and various clinicians is essential for adequate recognition of GP and the improvement of their psychophysical state. The Dutch research group further investigated the occurrence of GP. Among 1926 respondents 45.5\% reported concurrent genital involvement. Males predominated in this population (53.3\% vs $38.2 \%$ ), which was explained by easier inspection of male external genitalia compared to females. In most patients the age of onset of GP was between 20 and 40 [3].

\section{SYMPTOMS}

The genital skin is very sensitive and fragile to mechanical as well as chemical irritants. The most common irritants 
are: tampons, sanitary pads, tight clothes, shaving, vaginal discharge, sweat, semen, urine, faeces, bath cosmetics and soap, lubricants, spermicides. The most characteristic symptom of GP is itch of genital skin. While taking patient's history it is relevant to ask in detail about psoriasis in the family, sexual activity including recent partners along with other skin lesions in other areas. Psoriatic lesions at the genitalia present mostly as well-demarcated, brightly erythematous, thin plaques without scaling (Fig. 1), which is typical in other areas because of maceration [4]. If scales are present, they are observed only on more keratinised parts of the genital skin [5]. In addition to plaque-type genital psoriasis, the genital area may also be involved in pustular psoriasis [6]. In female patients vulvar psoriasis is mostly symmetrical and can vary from moist greyish plaques or glossy red plaques with no scales in the skin folds to silvery, scaling patches adjoining to the outer parts of the labia majora [7]. In males genital psoriatic lesions may be located on both scrotal and penile skin. The glans penis is the area of genital skin that is most commonly affected. Typically, GP does not involve the entire penis, scrotum, and inguinal folds [8]. Usually, in uncircumcised male patients well-defined non-scaling plaques are present under the prepuce and on the proximal glans, while in circumcised males red lesions are present on the glans and corona [9]. Genital psoriasis is considered a variety of the flexural (inverse) psoriasis, with the perineal, and axillary flexures affected instead of the typical localization of plaques [10].

The diagnosis of GP is usually based on clinical examination. Since psoriatic patients may present to a variety of specialists and GP is considered to be an embarrassing health condition, it is recommended to always ask about any genital complaints and examine the genital area in such patients. Dermatitis, candidiasis, squamous cell carcinoma, plasma-cell balanitis or vulvitis, lichen planus, syphilis,

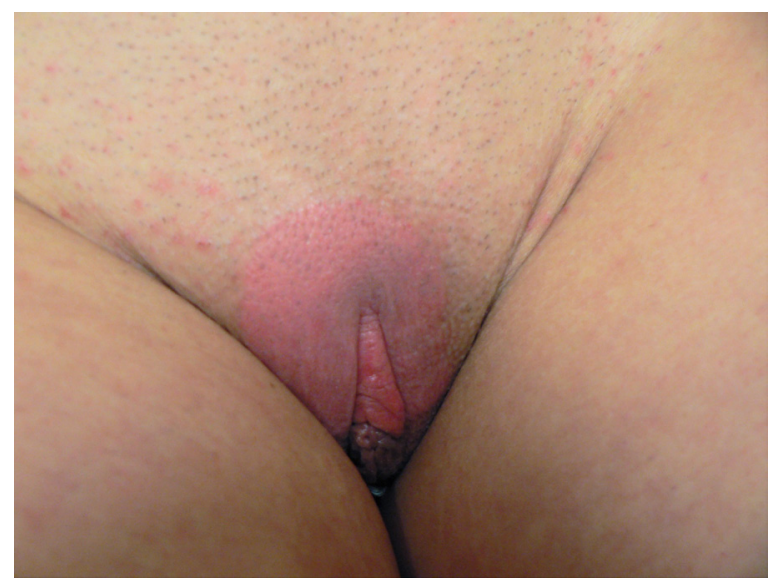

Figure 1. Psoriatic lesions on the female genitalia - welldemarcated, brightly erythematous, thin plaques without scaling

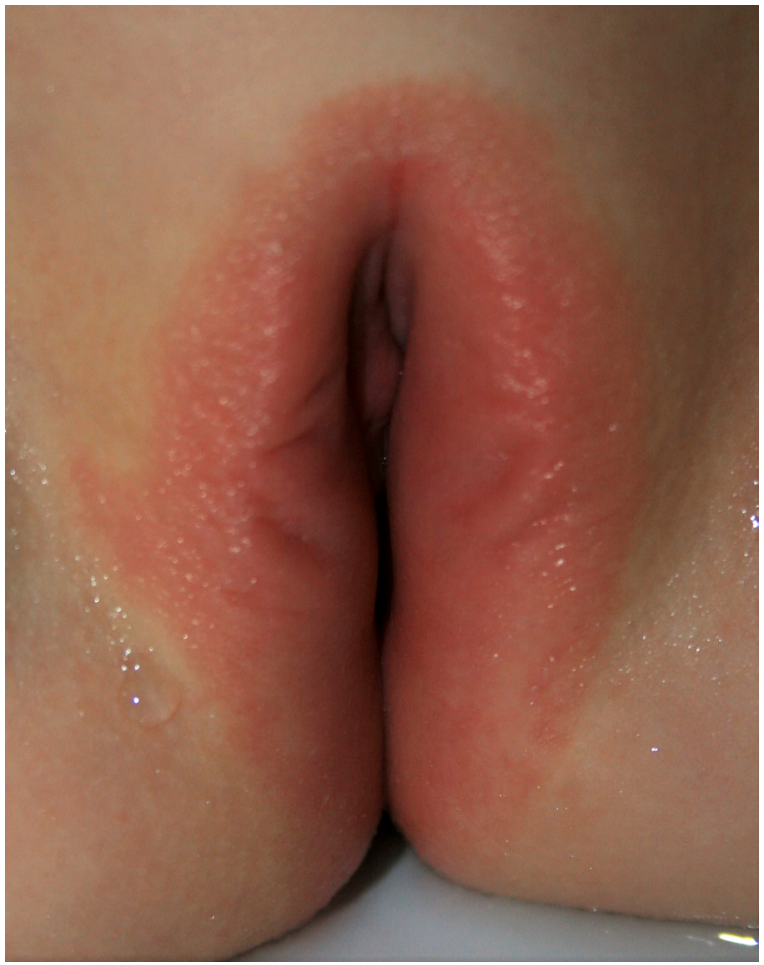

Figure 2. Genital psoriasis in a prepubertal girl. Vulvar psoriasis is symmetrical with glossy red plaques and no scales in the skin folds

scabies and pediculosis pubis are conditions taken into consideration during differential diagnosis of genital skin changes [11]. GP is not exclusive for adults, it may also be seen in younger patients (Fig. 2). There are many similarities between adult and paediatric genital dermatoses, however, streptococcal vulvovaginitis appears only at prepuberty, while candidiasis at postpuberty [12]. According to Fischer, the vulvar presentation of psoriasis is more common in children than in adults [13]. The need to search for nail pitting, scalp or postauricular erythema and scaling during paediatric clinical examination was also emphasized [13]. In a Clinical Audit of 130 cases of vulvar disease in children the authors reported that psoriasis is one of the most common dermatoses in prepubertal girls, but it may involve any age group including infants ("napkin psoriasis") [13-14]. Vulva itch and rashes are usually the symptoms presented to a paediatrician or general practitioner. The possibility of sexual abuse should not be omitted, especially when the child presents symptoms of a sexually transmitted disease or evidence of trauma is seen [14].

\section{QUALITY OF LIFE}

Psoriasis, especially the genital manifestation of the disease, has a great negative influence on patients' quality of life. The treatment is long-term and is inconvenient for patients because of required regularity and strict compliance to medication regimens. Finlay et al. stated that more 
than $80 \%$ of psoriatic patients report problems with social contacts and interpersonal relations. Personal life is particularly afflicted by genital psoriasis [15]. Psoriasis influences not only physical health, but psychosocial and emotional as well. Psoriasis deteriorates general wellbeing to a greater extent than most chronic diseases such as diabetes or cardiovascular disease [10]. Skin lesions, especially in the genital area and on the face may lead to severe depression and even to suicidal ideation. In Gupta's study almost 10\% of subjects reported a wish to be dead while $5.5 \%$ presented active suicidal ideation [16]. Supporting regular pharmacologic therapy with psychotherapy results in much better management of psoriasis and reduction of stress. Integrated treatment results in significant improvement in clinical severity of psoriasis. Stress is reported as a triggering factor of psoriasis by $60 \%$ of patients [17]. A study on the quality of life and sexual life in 487 patients with GP concluded that:

- patients with genital lesions report even significantly worse quality of life than patients without genital lesions;

- sexual distress and dysfunction are particularly prominent in women;

- sexual distress is especially high when genital skin is affected;

- the attention given to possible sexual problems in the psoriasis population by healthcare professionals is perceived as insufficient by patients [18].

A recent report confirmed the abovementioned statements and specified that sexual dysfunction and distress are prominent in approximately half of women with psoriasis [19]. Meeuwis et al. investigated which factors decrease the sexual activity of psoriatic patients. The main ones influencing distress and dysfunction scores were shame and embarrassment about physical appearance, lower sexual desire of the patient, annoyance of skin scaling and inconvenience of topical therapy [18]. The authors also concluded that women's quality of sexual life is more highly influenced by stress levels [18]. The course of the disease may vary during pregnancy: $55 \%$ of the patients reported improvement, $18 \%$ demonstrated steady course and $24 \%$ noticed worsening of psoriasis [20]. Remission of psoriasis during pregnancy is probably a result of hormonal levels changes such as estrogen, progesterone and glucocorticoids. Th2 based immune response might also be important for improvement of patients' condition [21]. The connection between psoriasis and pregnancy complications had also been investigated. A significant correlation between psoriasis and pregnancy complications such as miscarriage, chronic hypertension and perinatal complications and preterm delivery had been shown [22]. Pregnancy itself may influence self-esteem and result in lack of acceptance of changing appearance. Additional stress connected with exposure of genital psori- atic alterations during regular gynaecological examination can trigger depression and worsen the symptoms of the disease [23]. After delivery $62-87 \%$ of women developed new lesions in less than 2 months [24]. There is no reported correlation between foetus sex or breastfeeding and psoriasis exacerbation [25].

\section{TREATMENT}

Treatment of the sensitive genital skin is challenging, because of the unique genital environment (warmth, moisture and exposure to friction) [26]. Moreover, a suitable choice of remedies is necessary to avoid irritation in this area. The issue is further complicated due to paucity of evidence-based data on the efficacy and safety of available treatment options, originating mostly from individual case reports and case series [11]. Aside from genital lesions coexisting with severe and extensive psoriasis, treatment is generally topical. Topical corticosteroids are the most commonly recommended therapeutic option in GP. Owing to increased penetration of these agents in genital area and the resultant side effects, most authors recommend long-term use of weak to moderate corticosteroids; however, these are often not potent enough to induce a response. Others advocate short-term or intermittent administration of moderate to potent corticosteroids, or their use as an induction therapy followed by maintenance treatment with weaker preparations. Any treatment with topical steroids in the genital region should be individualized. Universal treatment regimens are not available. It is accepted that due to possible adverse effects and tachyplaxis the treatment should not last more than 2-4 weeks. Approved steroid preparations include $1 \%-2.5 \%$ hydrocortisone cream (class I - mild), $0.1 \%$ hydrocortisone butyrate cream (class I), fluticasone propionate (class II - moderate), mometasone furoate ointment (class III — potent) [27]. Sticherling recommends treatment with moderately potent steroids e.g. methylprednisolone aceponate (class I) and mometasone (class III) or alternatively bethametasone (class II/III) [28]. In severe and recalcitrant cases clobetasol propionate $0.05 \%$ may be applied daily but not longer than for 2-4 weeks (class IV - very potent). Some authors suggest that bethametasone should be used as a first line drug in genital lesions. If no significant response is observed within 7-10 days, it is recommended to increase dosage or potency of topical steroids [27]. Another option are mild topical tar preparations and topical vitamin D analogues. Since these agents may cause irritation of the sensitive genital skin, they are usually prescribed in combination with topical corticosteroids, rather than in monotherapy [29]. Also topical treatment with immunomodulators, pimecrolimus ointment or tacrolimus cream, was recommended in monotherapy or in combination with weak corticosteroids 
[30-32]. However, it should be remembered that besides irritation, the immunomodulating agents may promote allergic dermatitis, candidiasis and (re)activation of viral infections [11]. In patients with suspected opportunistic bacterial or fungal infections topical antibiotics or ketoconazole/miconazole should be implemented to prevent the Koebner effect [11]. Conservative treatment of genital psoriasis includes use of mild emollients and avoiding exposure to local irritating factors [33]. Phototherapy and laser therapy are not recommended in the genital area [34]. In sporadic cases, complete remission of genital psoriasis was documented after four weeks of treatment with oral dapsone (100 mg once a day) $[6,26]$. Psoriasis treatment in pregnant women is a challenge and both mother's and foetal health should be taken into consideration, when choosing a drug regimen. Despite being classified as $C$ category by the FDA, mild to moderate topical corticosteroids with emollients are the first line treatment in pregnant patients with psoriasis. In second line treatment UVB is used, while cyclosporine and biological therapy with anti-TNF-a are recommended as third line [35]. Unfortunately, patients commonly do not use prescribed drugs adequately, and this has a negative impact on treatment results. The low compliance was mostly caused by lack of satisfactory efficacy, unpleasant cosmetics components, time consumption and side effects [2]. Moreover, adverse reactions resulting in lower quality of life, such as methotrexate induced sexual disturbances and impotence, were the reasons why many psoriatic patients discontinued therapy. Genital psoriasis is an embarrassing disease for most patients, who usually do not know where to find professional advice and are ashamed of reporting the ailments. Little social awareness often leads to confusing genital psoriasis with sexually transmitted diseases. Nevertheless, patients believe that insufficient attention is given to their sexual problems while seeking medical advice. Before presenting to a specialist, especially young patients try to treat genital psoriasis with domestic remedies and such behaviour usually exacerbates symptoms and patients' condition. Genital psoriasis is not only a dermatological problem and for that reason consultations with other specialists are recommended for better treatment efficacy. General practitioners and paediatricians play a significant role in the initial recognition of the disease. Furthermore, dermatologists, gynaecologists and urologists' close cooperation is required to achieve a satisfactory outcome in genital psoriasis treatment. Proper communication with the patient together with holistic treatment increases compliance and results in greater quality of life improvements. Involving a sexologist in the management of genital psoriasis has a significant, positive implication on patients' wellbeing. It was shown in the Dutch study of genital psoriasis aware- ness programme, that a vast majority of patients obtained prompt improvement, when treated by a multidisciplinary team of specialists [19].

\section{CONCLUSIONS}

Genital psoriasis affects both general and sexual quality of life, which was demonstrated in various studies. Genital lesions influence female sexual life more significantly, but both males and females achieved lower scores in quality of life questionnaires in comparison to healthy cohorts. To improve the efficacy of treatment a multidisciplinary team of clinicians should be involved in all stages of diagnosis and treatment. Patient's psychosocial life aspects are of the great importance in case of GP. Discussing all aspects of patients' wellbeing leads to earlier diagnosis, adequate treatment and better compliance.

\section{Conflict of interest}

There is no specific funding to declare.

\section{REFERENCES}

1. Icen M, Crowson CS, McEvoy MT, [et al.]. Trends in incidence of adult-onset psoriasis over three decades: a population-based study. J Am Acad Dermatol. 2009, 60, 394-401.

2. Fouéré $\mathrm{S}$, Adjadj L, Pawin H. How patients experience psoriasis: results from a European survey. J Eur Acad Dermatol Venereol. 2005, 19, 2-6.

3. Meeuwis KAP, de Hullu JA, de Jager ME, [et al.]. Genital psoriasis: a questionnaire-based survey on a concealed skin disease in the Netherlands. J Eur Acad Dermatol Venereol. 2010, 24, 1425-1430.

4. Rosen T. Update on genital lesions. JAMA. 2003, 290, 1001-1005.

5. Varghese $M$, Kindel S. Pigmentary disorders and inflammatory lesions of the external genitalia. Urol Clin North Am. 1992, 19, 111-121.

6. Singh N, Thappa DM. Circinate pustular psoriasis localized to glans penis mimicking 'circinate balanitis' and responsive to dapsone. Indian J Dermatol Venereol Leprol. 2008, 74, 388-389.

7. Welsh BM, Berzins KN, Cook KA, [et al.]. Management of common vulval conditions. Med J Aust. 2003, 178, 391-395.

8. Bonnetblanc JM. Psoriasis. Ann Dermatol Vnéréologie. 2006, 133, 298-299.

9. Van Dijk F, Thio HB, Neumann HAM. Non-Oncological and Non-Infectious Diseases of the Penis (Penile Lesions). EAU-EBU Updat Ser. 2006, 4, 13-19.

10. Langley RGB, Krueger GG, Griffiths CEM. Psoriasis: epidemiology, clinical features, and quality of life. Ann Rheum Dis. 2005, 64, 18-25.

11. Meeuwis KAP, de Hullu JA, Massuger LFG, [et al.]. Genital psoriasis: A systematic literature review on this hidden skin disease. Acta Derm Venereol. 2011, 91, 5-11.

12. Welsh B, Howard A, Cook K. Vulval itch. Aust Fam Physician. 2004, 33, 505-510.

13. Fischer GO. Vulval disease in pre-pubertal girls. Australas J Dermatol. 2001, 42, 225-234.

14. Fischer G, Rogers M. Vulvar disease in children: a clinical audit of 130 cases. Pediatr Dermatol. 2000, 17, 1-6.

15. Finlay AY, Coles EC. The effect of severe psoriasis on the quality of life of 369 patients. Br J Dermatol. 1995, 132, 236-244.

16. Gupta MA, Schork NJ, Gupta AK, [et al.]. Suicidal ideation in psoriasis. Int J Dermatol. 1993, 32, 188-190.

17. Seville RH. Psoriasis and stress. Br J Dermatol. 1977, 97, 297-302.

18. Meeuwis KAP, de Hullu JA, van de Nieuwenhof HP, [et al.]. Quality of life and sexual health in patients with genital psoriasis. Br J Dermatol. 2011, 164, 1247-1255.

19. Meeuwis KAP, De Hullu JA, Inthout J, [et al.]. Genital Psoriasis Awareness Program : Physical and Psychological Care for Patients with Genital Psoriasis. Acta Derm Venereol. 2015, 95, 211-216.

20. Raychaudhuri S, Navare T, Gross J, [et al.]. Clinical course of psoriasis during pregnacy. Int J Dermatol. 2003, 42, 518-520. 
21. Hoffman MB, Farhangian M, Feldman SR. Psoriasis during pregnancy: characteristics and important management recommendations. Expert Rev Clin Immunol. 2015, 11, 709-720.

22. Makara-Studzińska M, Pietrzak A, Lewicka M, [et al.]. Somatic and non-somatic problems connected with psoriasis in pregnancy. Ginekol Pol. 2013, 84, 211-213.

23. Pietrzak A, Janowski K, Lechowska-Mazur I. Psoriasis is a chronic skin disease in the context of psychology. New Med. 2006, 1, 14-19.

24. Boyd AS, Morris LF, Phillips CM, [et al.]. Psoriasis and pregnancy: hormone and immune system interaction. Int J Dermatol. 1996, 35, 169-172.

25. Dąbrowska-Członka M, Chodorowska G, Bartosińska J. Przebieg łuszczycy w ciąży. Nowa Medycyna. 2006, 1, 1-3.

26. Guglielmetti A, Conlledo R, Bedoya J, [et al.]. Inverse psoriasis involving genital skin folds: successful therapy with dapsone. Dermatol Ther (Heidelb). 2012, 2, 15.

27. Moyal-Barracco M, Wendling J.Vulvar dermatosis. Best Pract and Res Clin Obstet and Gynaecol. 2014, 28, 946-958.

28. Sticherling M. Vulvar psoriasis. In: Kirtschig G and Cooper SM (eds.). Gynecologic dermatology, symptoms, signs and clinical management. JP Medical Ltd., London 2016, 109-114.
29. Menter A, Korman NJ, Elmets CA, [et al.]. Guidelines of care for the management of psoriasis and psoriatic arthritis: section 6. Guidelines of care for the treatment of psoriasis and psoriatic arthritis: case-based presentations and evidence-based conclusions. J Am Acad Dermatol. 2011, 65, 137-174.

30. Rallis E, Nasiopoulou A, Kouskoukis C, [et al.]. Successful treatment of genital and facial psoriasis with tacrolimus ointment $0.1 \%$. Drugs Exp Clin Res. 2005, 31, 141-145.

31. Trager JDK. What's your diagnosis? Well-demarcated vulvar erythema in two girls. J Pediatr Adolesc Gynecol. 2005, 18, 43-46.

32. Bissonnette R, Nigen S, Bolduc C. Efficacy and tolerability of topical tacrolimus ointment for the treatment of male genital psoriasis. J Cutan Med Surg. 2008, 12, 230-234.

33. Edwards SK, Bates $C M$, Lewis $F$, [et al.]. UK national guideline on the management of vulval conditions. Int J STD AIDS. 2015, 26, 611-624.

34. Menter A, Korman NJ, Elmets CA, [et al.]. Guidelines of care for the management of psoriasis and psoriatic arthritis: Section 5. Guidelines of care for the treatment of psoriasis with phototherapy and photochemotherapy. J Am Acad Dermatol. 2010, 62, 114-135.

35. Bangsgaard N, Rørbye C, Skov L. Treating Psoriasis During Pregnancy: Safety and Efficacy of Treatments. Am J Clin Dermatol. 2015, 16, 389-398. 